\title{
Percutaneous reduction of irreducible dislocation of the interphalangeal joint of the hallux: A simple technique for a rare injury
}

\author{
Erdi Özdemir, MD®, Ali Teoman Evren, MD®D, Mehmet Özer, MD®, Murat Altay, MD 1 \\ Department of Orthopedics and Traumatology, University of Health Sciences, Ankara Keçiören Training and Research Hospital, Ankara, Turkey
}

Irreducible dislocation of the interphalangeal joint (IPJ) of hallux is an uncommon injury due to the inherent stability of the joint. ${ }^{[1]}$ Invagination of the sesamoid-plantar plate complex into the interphalangeal space is the main reason for unsuccessful closed reduction attempts. ${ }^{[2]}$ Open reduction is the commonly used technique in the literature following unsuccessful closed reduction of the IPJ. ${ }^{[3,4]}$ However, percutaneous reduction of the dislocated IPJ could be an option. There are only two articles in the literature reporting different percutaneous reduction techniques for the irreducible dislocation of the IPJ of the hallux. ${ }^{[5,6]}$ In this article, Kirschner (K)-wire assisted percutaneous reduction of incarcerated sesamoid was performed, confirming satisfactory reduction of dislocation. ${ }^{[7,8]}$

Received: June 02, 2020

Accepted: June 25, 2020

Published online: September 11, 2020

Correspondence: Erdi Özdemir, MD. SBÜ Keçiören Eğitim ve Araştırma Hastanesi Ortopedi ve Travmatoloji Kliniği, 06280 Keçiören, Ankara, Türkiye.

E-mail: erdi.ozdemir@hacettepe.edu.tr

Doi: 10.5606/ehc. 2020.76937

Citation: Özdemir E, Evren AT, Özer M, Altay M. Percutaneous reduction of irreducible dislocation of the interphalangeal joint of the hallux: A simple technique for a rare injury. Jt Dis Relat Surg 2020:31(3):610-613.

(2020 All right reserved by the Turkish Joint Diseases Foundation

This is an open access article under the terms of the Creative Commons Attribution-NonCommercial License, which permits use, distribution and reproduction in any medium, provided the original work is properly cited and is not used for commercial purposes (http://creativecommons.org/licenses/by-nc/4.0/).

\section{ABSTRACT}

Irreducible dislocation of the interphalangeal joint (IPJ) of hallux is a rare injury due to its intrinsic stabile anatomy. Open reduction is the commonly preferred treatment option once closed reduction attempts fail. In this article, we present a 37-year-old male patient with an irreducible dislocation of the IPJ of the hallux treated with Kirschner (K)-wire assisted percutaneous reduction. The patient was symptom free at the sixth month of follow-up. In conclusion, $\mathrm{K}$-wire assisted percutaneous reduction might be an option in the treatment of irreducible dislocation of IPJ of the hallux.

Keywords: Dislocation, great toe, incarcerated sesamoid, interphalangeal joint, minimal invasive.

\section{CASE REPORT}

A 37-year-old male patient was admitted to the emergency department after a wood block fell on his right foot. He was complaining of pain, swelling, and tenderness over the right hallux. The physical examination revealed marked swelling over the right hallux while an obvious deformity was not observed owing to the swelling. The subdermal venous plexus refill time was normal and no neurologic deficit was detected. He had no external wounds. Plain radiographs demonstrated the dislocation of the IPJ of right hallux. In addition, sesamoid was observed as entrapped within the IPJ (Figure 1). Closed reduction was performed at the emergency room. However, the IPJ could not be reduced successfully. The IPJ dislocation was considered as Miki type I irreducible dislocation of the IPJ of hallux ${ }^{[2]}$ and we decided to perform percutaneous reduction of the IPJ. A written informed consent was obtained from the patient.

The right hallux was anesthetized with a digital block using $2 \%$ prilocaine. A stab incision was performed on the dorsomedial side of the IPJ not to 


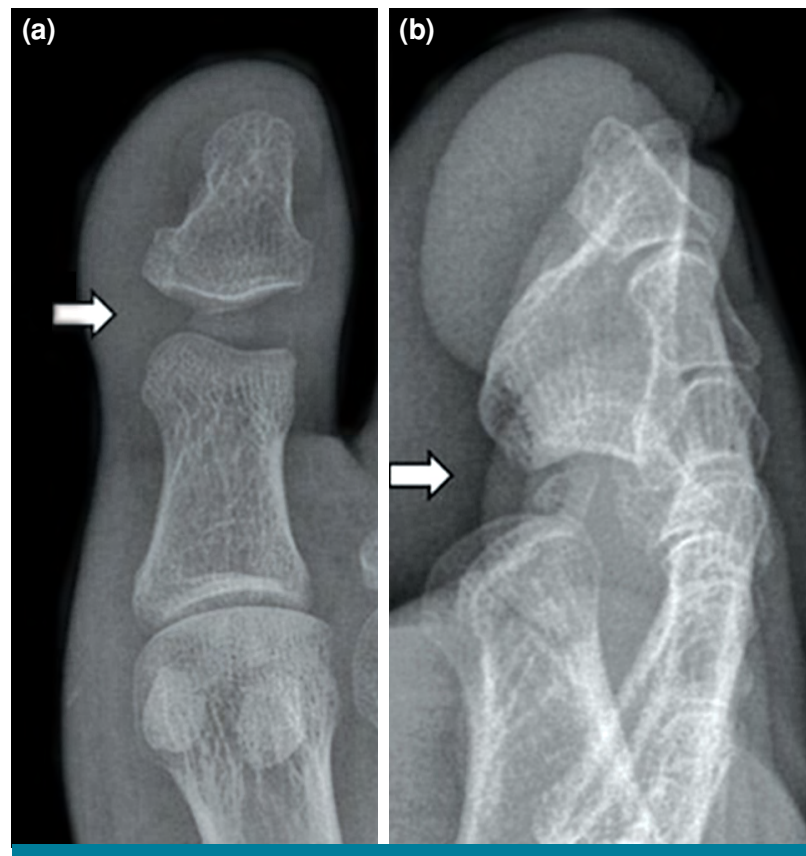

FIGURE 1. (a) Anteroposterior and (b) lateral radiographs demonstrating irreducible dislocation of interphalangeal joint of hallux, sesamoid was entrapped within joint.

harm the extensor hallucis longus (EHL) tendon. Then, the blunt side of a $1.5 \mathrm{~mm}$ K-wire was introduced into the IPJ through the incision (Figure 2a). The sesamoid-plantar plate complex was pushed with the blunt side of the K-wire by hand under fluoroscopic guidance (Figure 2b, Figure 3). After several attempts, sesamoid-plantar plate complex was reduced to its original location. Final reduction was evaluated by fluoroscopy confirming that the sesamoid was repositioned in plantar aspect of the IPJ. However, the IPJ was clinically unstable thus temporary arthrodesis was performed to the IPJ with a K-wire (Figure 4). The patient was allowed to heel touch after the operation and followed up at first, third and sixth weeks. Tenderness and pain were improved at postoperative second day. Swelling was almost disappeared at first week of follow-up. At third week of follow-up, the patient could walk on heel touch almost pain-free. At sixth week of follow-up, radiographs of his right IPJ showed good alignment with congruent joint (Figure 5) thus K-wire was removed. At sixth month of follow-up, the patient had mild stiffness on the IPJ; however, he was able to walk without pain and returned to his previous activity level.

\section{DISCUSSION}

Due to natural stability, irreducible IPJ dislocation of the hallux is a rare injury. There are several intrinsic stabilizer structures around the joint including dorsal capsule, sesamoid-plantar plate, medial and lateral collateral ligaments, extensor and flexor hallucis longus tendons. Besides, the condylar shape of IPJ also contributes to stability. ${ }^{[1]}$ Unfortunately, this anatomy may make closed reduction difficult and sometimes even impossible after dislocation of the IPJ.

Interphalangeal sesamoids are embedded within the joint capsule at the plantar aspect of the hallux
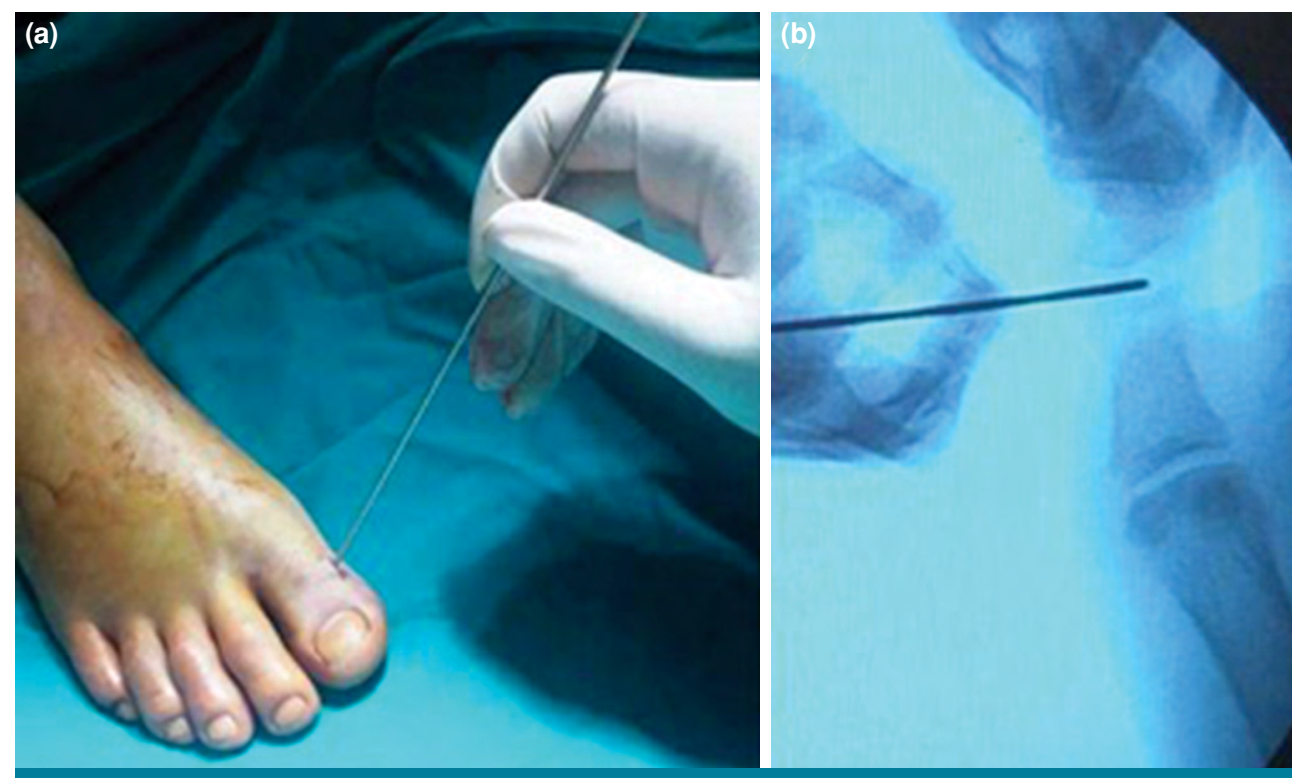

FIGURE 2. (a) A stab incision performed on dorsomedial side of joint. (b) Sesamoid-plantar plate complex was pushed with blunt side of K-wire by hand under fluoroscopic guidance. 


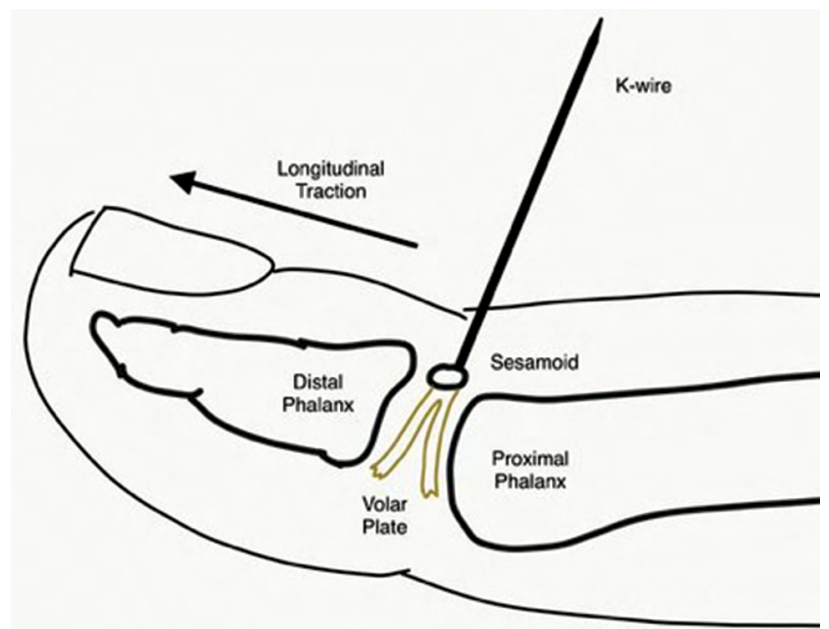

FIGURE 3. An illustration demonstrating percutaneous reduction technique. Blunt side of a $1.5 \mathrm{~mm} \mathrm{~K}$-wire was introduced into interphalangeal joint of hallux through incision performed on dorsomedial side of joint. Sesamoid-plantar plate complex was pushed gently with blunt side of K-wire by hand until sesamoid-plantar plate complex was reduced to its original location.

and ossified sesamoid may alter biomechanics and limit joint movement. The prevalence of ossified sesamoids has been reported variably at $2-13 \% .{ }^{[9]}$ The sesamoid of IPJ is invisible radiographically at $44 \%$ of the IPJ dislocation cases which could mislead the diagnosis of sesamoid incarceration and complicate the confirmation of successful reduction. ${ }^{[2]}$

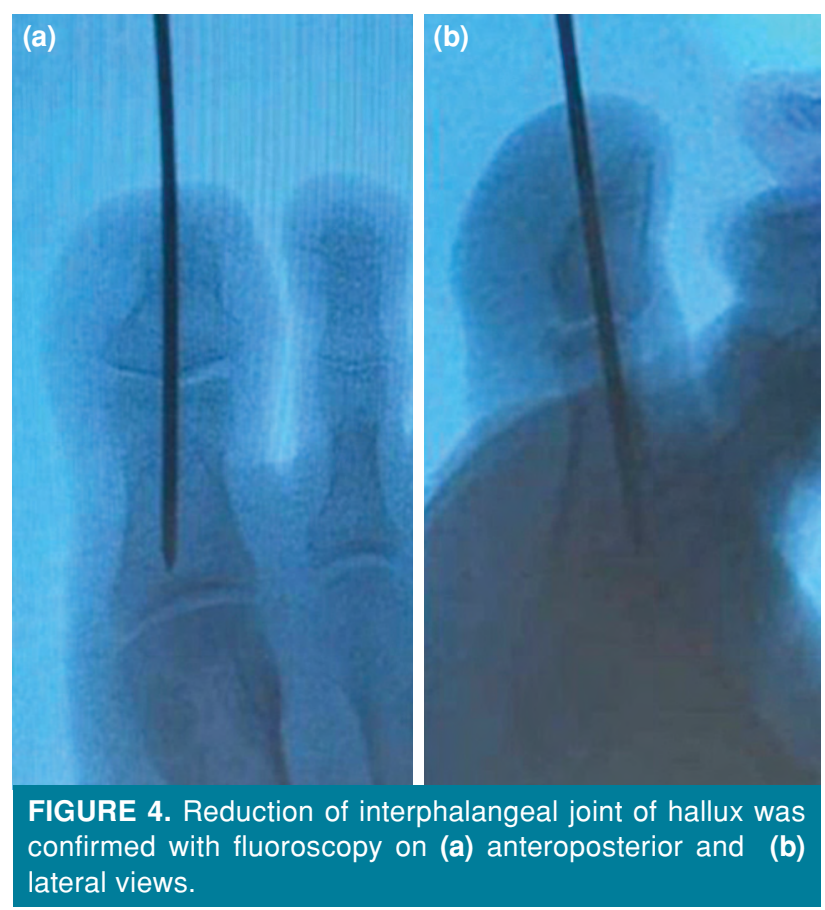

Miki et al. ${ }^{[2]}$ classified dorsal irreducible IPJ dislocations into two types based on sesamoid and volar plate configuration. In Miki type I injuries, volar plate is detached from proximal attachment on the proximal phalanx resulting in incarceration of both sesamoid and volar plate into the IPJ, which makes hallux slightly elongated, widens joint space but maintains correct alignment. In Miki type II injuries, volar plate is completely detached from the proximal and distal attachments and completely displaced which causes sesamoid bone overriding of the proximal phalanx head, narrowing of joint space and sometimes even overlapping of phalanges and hyperextension of the distal phalanx. ${ }^{[2]}$

The mechanism of the IPJ dislocation is mostly a hyperextension and abduction injury. Once the IPJ joint of the hallux is dislocated, plantar plate could invaginate into the joint. Since the collateral ligaments are still tight and the plantar plate is locked within the joint, manual manipulation and reduction are challenging after dislocation of the IPJ. ${ }^{[10]}$ During the closed reduction, the IPJ is dorsiflexed under longitudinal traction and the deformity is exaggerated. Then, a gentle plantar flexion is applied as the longitudinal traction continues. This maneuver may produce a click sound indicating the reduction of the IPJ. ${ }^{[11]}$ Instability and re-dislocation are common problems following closed reduction. Frequently, temporary arthrodesis of the IPJ with a K-wire is required. ${ }^{[10]}$ Our patient had clinical instability after
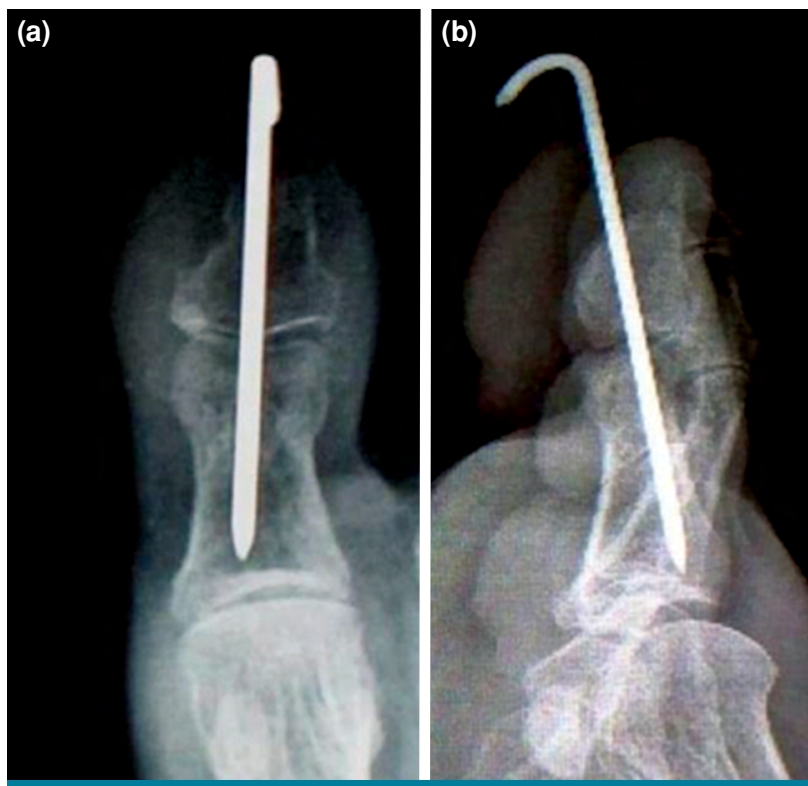

FIGURE 5. (a) Anteroposterior and (b) lateral radiographs of interphalangeal joint of hallux showed good alignment with congruent joint at sixth week of follow-up. 
the reduction of the IPJ so that we performed a temporary arthrodesis of IPJ with a K-wire.

To the best of our knowledge, the first case of irreducible dislocation of the IPJ of the hallux was reported in 1944. The patient presented with an incarcerated sesamoid and treated with open reduction. ${ }^{[12]}$ Since then, open reduction has been commonly used for irreducible IPJ dislocation of the hallux if closed reduction fails..$^{[1,3,10,13-15]}$ Arthroscopic reduction of the irreducible IPJ dislocation of the hallux has been reported. ${ }^{[16]}$ However, it is technically demanding and has no clear advantage compared to fluoroscopy-guided percutaneous surgery regarding soft tissue protection. ${ }^{[17]}$ Woon $^{[5]}$ described a novel percutaneous reduction technique for irreducible IPJ dislocation of the hallux in 2010. Since then, only one paper has been published by Takeda et al. ${ }^{[6]}$ utilizing a different percutaneous technique for irreducible IPJ dislocation of the hallux. Woon ${ }^{[5]}$ performed a stab incision over EHL tendon and maintained reduction with a K-wire introduced through the EHL tendon. Takeda et al. ${ }^{[6]}$ introduced an 18-gauge needle through the EHL tendon toward the invaginated sesamoid under longitudinal traction of the distal phalanx and achieved reduction without an incision. We have performed K-wire assisted percutaneous reduction with a stab incision performed on the dorsomedial side of the joint not to jeopardize the EHL tendon.

Percutaneous reduction technique is advantageous over open reduction since EHL tendon, dorsal capsule and surrounding soft tissue are not disrupted during the procedure. ${ }^{[5,6,18]}$ However, percutaneous reduction technique could be used in selected patients. It is not a feasible technique if the sesamoid is not visible radiographically since fluoroscopic confirmation is mandatory. Also, it is not recommended in chronic dislocations, open dislocations, and multiple trauma patients. ${ }^{[17]}$

In conclusion, K-wire assisted percutaneous reduction is a relatively harmless and less technically demanding technique compared to open and arthroscopic techniques. K-wire assisted percutaneous reduction may be an option in selected patients with irreducible dislocation of IPJ of the hallux if closed reduction fails.

\section{Declaration of conflicting interests}

The authors declared no conflicts of interest with respect to the authorship and/or publication of this article.

\section{Funding}

The authors received no financial support for the research and/or authorship of this article.

\section{REFERENCES}

1. Alexander L. Irreducible Traumatic Miki Type II Dislocation of Hallucal Interphalangeal Joint: An Insight into this Rare Injury 2019;13:PD01-PD03.

2. Miki T, Yamamuro T, Kitai T. An irreducible dislocation of the great toe. Report of two cases and review of the literature. Clin Orthop Relat Res 1988;230:200-6.

3. Imao K, Miwa H, Watanabe K, Imai N, Endo N. Dorsalapproach open reduction for irreducible dislocation of the hallux interphalangeal joint: A case series. Int J Surg Case Rep 2018;53:316-21.

4. Crosby LA, McClellan JW 3rd, Prochaska VJ. Irreducible dorsal dislocation of the great toe interphalangeal joint: case report and literature review. Foot Ankle Int 1995;16:559-61.

5. Woon CY. Dislocation of the interphalangeal joint of the great toe: is percutaneous reduction of an incarcerated sesamoid an option?: A report of two cases. J Bone Joint Surg [Am] 2010;92:1257-60.

6. Takeda S, Nishimura A, Yamaji S, Tabuchi A, Sudo A, Hirata H. Percutaneous reduction of a dislocation of the interphalangeal joint of the great toe: A case report. J Foot Ankle Surg 2020:S1067-2516(20)30089-2.

7. Atik OŞ. Is there something new and interesting in my article? Eklem Hastalik Cerrahisi 2019;30:69.

8. Atik OŞ. Which articles do we prefer to publish? Eklem Hastalik Cerrahisi 2018;29:1.

9. Nwawka OK, Hayashi D, Diaz LE, Goud AR, Arndt WF 3rd, Roemer FW, et al. Sesamoids and accessory ossicles of the foot: anatomical variability and related pathology. Insights Imaging 2013;4:581-93.

10. Yang IB, Sun KK, Sha WL, Yu KS, Chow YY. Interphalangeal dislocation of toes: a retrospective case series and review of the literature. J Foot Ankle Surg 2011;50:580-4.

11. Stienstra JJ, Derner R. Closed reduction of a proximal interphalangeal joint dislocation. J Foot Surg 1990;29:385-7.

12. Müller GM. Dislocation of sesamoid of hallux. Lancet 1944;243:789.

13. Wolfe J, Goodhart C. Irreducible dislocation of the great toe following a sports injury. A case report. Am J Sports Med 1989;17:695-6.

14. Dave D, Jayaraj VP, James SE. Intra-articular sesamoid dislocation of the interphalangeal joint of the great toe. Injury 1993;24:198-9.

15. Bin Abd Razak HR, Chia ZY, Tan HC. Irreducible dislocation of the great toe interphalangeal joint secondary to an incarcerated sesamoid. Case Rep Orthop 2015;2015:231685.

16. Lui TH. Interphalangeal arthroscopy of the toes. Foot (Edinb) 2014;24:42-6.

17. Woon CY. Dislocation of the interphalangeal joint of the great toe: is percutaneous reduction of an incarcerated sesamoid an option? Surgical technique. J Bone Joint Surg [Am] 2011;93 Suppl 1:109-12.

18. Suwannahoy P, Srisuwan T, Pattamapaspong N, Mahakkanukrauh P. Intra-articular ossicle in interphalangeal joint of the great toe and clinical implication. Surg Radiol Anat 2012;34:39-42. 